\title{
Bridging thermodynamics and metrology in nonequilibrium quantum thermometry
}

\author{
Vasco Cavina, ${ }^{1}$ Luca Mancino, ${ }^{2}$ Antonella De Pasquale, ${ }^{3,4,1}$ Ilaria Gianani, ${ }^{2}$ Marco Sbroscia, ${ }^{2}$ Robert I. Booth, $, 2,5$ \\ Emanuele Roccia, ${ }^{2}$ Roberto Raimondi, ${ }^{6}$ Vittorio Giovannetti, ${ }^{1}$ and Marco Barbieri ${ }^{2}, 7$ \\ ${ }^{1}$ NEST, Scuola Normale Superiore and Istituto Nanoscienze-CNR, Piazza dei Cavalieri 7, I-56126 Pisa, Italy \\ ${ }^{2}$ Dipartimento di Scienze, Università degli Studi Roma Tre, Via della Vasca Navale 84, I-00146 Rome, Italy \\ ${ }^{3}$ Dipartimento di Fisica, Università di Firenze, Via G. Sansone 1, I-50019 Sesto Fiorentino (FI), Italy \\ ${ }^{4}$ INFN Sezione di Firenze, Via G. Sansone 1, I-50019 Sesto Fiorentino (FI), Italy \\ ${ }^{5}$ Institut de Physique, Sorbonne Université, 4 Place Jussieu, F-75005 Paris, France \\ ${ }^{6}$ Dipartimento di Matematica e Fisica, Università degli Studi Roma Tre, Via della Vasca Navale 84, I-00146 Rome, Italy \\ ${ }^{7}$ Istituto Nazionale di Ottica - CNR, Largo Enrico Fermi 6, I-50125 Florence, Italy
}

(Received 21 June 2018; revised manuscript received 7 September 2018; published 9 November 2018)

\begin{abstract}
Single-qubit thermometry presents the simplest tool to measure the temperature of thermal baths with reduced invasivity. At thermal equilibrium, the temperature uncertainty is linked to the heat capacity of the qubit, however, the best precision is achieved outside the equilibrium condition. Here, we discuss a way to generalize this relation in a nonequilibrium regime, taking into account purely quantum effects such as coherence. We support our findings with an experimental photonic simulation.
\end{abstract}

DOI: 10.1103/PhysRevA.98.050101

\section{INTRODUCTION}

Identifying strategies for improving the measurement precision by means of quantum resources is the purpose of quantum metrology [1-3]. In particular, through the quantum Cramér-Rao bound (QCRB), it sets ultimate limits on the best accuracy attainable in the estimation of unknown parameters even when the latter are not associated with observable quantities. These considerations have attracted increasing attention in the field of quantum thermodynamics, where an accurate control of the temperature is highly demanding [4-8]. Besides the emergence of primary and secondary thermometers based on precisely machined microwave resonators $[9,10]$, recent efforts have been made aiming at measuring temperature at even smaller scales, where nanosize thermal baths are highly sensitive to disturbances induced by the probe [11-17]. Some paradigmatic examples of nanoscale thermometry involve nanomechanical resonators [18], quantum harmonic oscillators [19], or atomic condensates [20-22] (also in conjunction with the estimation of the chemical potential [23]). In this context the analysis of quantum properties needs to be taken into account in order to establish, and eventually enhance, metrological precision [24-29].

In a conventional approach to thermometry, an external bath $\mathcal{B}$ at thermal equilibrium is typically indirectly probed via an ancillary system, the thermometer $\mathcal{S}$, that is placed into a weak interaction with the former. Assuming hence that the thermometer reaches the thermal equilibrium configuration without perturbing $\mathcal{B}$ too much, the Einstein theory of fluctuations (ETF) can be used to characterize the sensitivity of the procedure in terms of the heat capacity of $\mathcal{S}$ which represents its thermal susceptibility to the perturbation imposed by the bath [30-33]. Since the latter is an equilibrium property, one should not expect it to hold in nonequilibrium regimes. However, thermometry schemes that do not need a full thermalization of the probe have been recognized to offer higher sensitivities in temperature estimations [34], as also recognized by studies of the strong-coupling regime with the bath [35]. Thus, if, on the one hand, the QCRB can still be used as a proper tool to gauge the measurement uncertainty on the bath temperature, on the other hand, establishing a direct link between this approach and the thermodynamic properties of the probe is still an open question. Furthermore, the advantages pointed out in Ref. [34] are conditional on precisely addressing the probe during its evolution, a task which might be demanding in real experiments [28]. Here, $\mathcal{S}$ is assumed to be a quantum system characterized by a local Hamiltonian $H$ that, after being initialized into some proper input state $\rho(0)$, weakly interacts for some time $\tau$ with the bath $\mathcal{B}$ of the assigned, but unknown, temperature $T$, before been measured. In this setting, we compare the performances of optimal estimation procedures with standard thermometry approaches: The temperature parameter $T$ is recovered by only monitoring the energy variation on $\mathcal{S}$ by its interaction with the bath. Then we derive a universal inequality that links metrological and thermodynamic quantities, ultimately discussing the optimal condition for its saturation.

In particular, for the case where $\mathcal{S}$ is a two-level (qubit) system, we show that optimality can be achieved for a broad class of configurations that also include out-of-equilibrium scenarios. A quantum photonic experiment simulating the $\mathcal{S}-\mathcal{B}$ thermal coupling confirms our results, giving indications for optimal measurements for any platform.

\section{QCRB vs ETF}

In a conventional thermometry approach, the bath temperature is recovered by measuring the mean energy $E_{T}(\tau)=$ $\operatorname{tr}[H \rho(\tau)]$ of $\mathcal{S}$ and inverting its functional dependence upon $T, \rho(\tau)$ being the reduced density matrix describing the state of $\mathcal{S}$ after its interaction with $\mathcal{B}$. In the absence of an 
extra source of experimental noise, a simple application of the error propagation formula reveals that, in this scenario, the associated mean-square error (MSE) of the estimation procedure can reach the value

$$
\Delta^{2} T=\frac{\Delta^{2} E_{T}(\tau)}{M C_{T}^{2}(\tau)},
$$

where $M$ is the number of measurements one performs on the probe. In this expression, explicitly derived in the Supplemental Material [36], $\Delta^{2} E_{T}(\tau)=\operatorname{tr}\left\{\left[H-E_{T}(\tau)\right]^{2} \rho(\tau)\right\}$ is the variance of $H$ on $\rho(\tau)$ which we use to estimate the uncertainty associated with the mean energy measure, and $C_{T}(\tau)=\partial_{T} E_{T}(\tau)$ is the partial derivative of $E_{T}(\tau)$ with respect to $T$. The latter quantity represents the energetic susceptibility of the system to the perturbation imposed by the bath: Since energy is only exchanged in the form of heat, it coincides with its heat capacity (HC), even in and out of thermal equilibrium [30-32].

The accuracy level represented by (1) is arguably not the best for which one can hope. As a matter of fact, it cannot be excluded that different estimation procedures could be devised based on generalized positive-operator-valued measures (POVMs) on $\mathcal{S}$ which possibly allow one to better recover the value of $T$. The ultimate performances achievable by such procedures are set by the QCRB [2,3] which, irrespectively of the specific form of the probe-bath coupling, establishes the following inequality,

$$
\Delta^{2} T \geqslant \frac{1}{M Q_{T}(\tau)},
$$

where $Q_{T}(\tau)$ is the quantum Fisher information (QFI), a functional which only depends on $\rho(\tau)$-explicitly this is given by $Q_{T}^{(\tau)}=\operatorname{tr}\left[L_{T} \partial_{T} \rho(\tau)\right]$, with $L_{T}$ being the symmetric logarithmic derivative of the problem, i.e., the self-adjoint operator which satisfies the identity $\partial_{T} \rho(\tau)=1 / 2\left\{L_{T}, \rho(\tau)\right\}$, with $\{\cdots, \cdots\}$ being the anticommutator [3].

Applying (2) to (1), we can hence draw the following universal relation,

$$
Q_{T}(\tau) \geqslant \frac{C_{T}^{2}(\tau)}{\Delta^{2} E_{T}(\tau)},
$$

that links together the generalized $\mathrm{HC}$ of $\mathcal{S}$, its energy spread $\Delta^{2} E_{T}(\tau)$, and the associated QFI functional. Equation (3) is saturated when ETF holds, i.e., when $\tau$ is sufficiently long to guarantee the thermalization condition, i.e., when $\mathcal{S}$ reaches the equilibrium state represented by the thermal Gibbs state $\rho_{T}^{\text {(eq) }}=e^{-H / k_{B} T} / \mathcal{Z}$, with $\mathcal{Z}=\operatorname{tr}\left[e^{-H / k_{B} T}\right]$ the partition function of the system. In this scenario, in fact, one has [37,38] (see Supplemental Material [36])

$$
Q_{T}^{(\mathrm{eq})}=\frac{\Delta^{2} E_{T}^{(\mathrm{eq})}}{k_{B}^{2} T^{4}}, \quad C_{T}^{(\mathrm{eq})}=\frac{\Delta^{2} E_{T}^{(\mathrm{eq})}}{k_{B} T^{2}},
$$

which can be combined to give $Q_{T}^{(\mathrm{eq})}=\left[C_{T}^{(\mathrm{eq})}\right]^{2} / \Delta^{2} E_{T}^{(\mathrm{eq})}$. Accordingly, one can conclude that, when the thermometer and the bath reach thermal equilibrium, the standard thermometry procedure which derives $T$ from the mean energy of $\mathcal{S}$ is optimal. We point out that Eq. (4) also establishes a direct linear dependence between QFI and the associated capacity, i.e.,

$$
Q_{T}^{(\mathrm{eq})}=C_{T}^{(\mathrm{eq})} /\left(k_{B} T^{2}\right),
$$

which, as we shall clarify in the following, is a peculiar property of Gibbs states.

\section{THE QUBIT MODEL}

Let us now focus on the special case where the probe system $\mathcal{S}$ is a qubit, with a specific Hamiltonian $H=\hbar \omega \sigma_{z} / 2$, and $\mathcal{B}$ is a bosonic thermal bath (hereafter $\sigma_{z}$ being the third Pauli operator). As in Refs. [15,17-19,34] we describe the temporal evolution of $\mathcal{S}$ by assigning a Markovian master equation (MME), which we write in the interaction picture representation as $\dot{\rho}(t)=\sum_{j= \pm} \gamma_{j} \mathcal{D}_{j}[\rho(t)]$. In this expression, $\mathcal{D}_{-}$and $\mathcal{D}_{+}$are Gorini-Kossakowski-SudarshanLindblad (GKSL) generators having, respectively, the qubit ladder matrices $\sigma_{-}=|0\rangle\langle 1|$ and $\sigma_{+}=|1\rangle\langle 0|$ as corresponding Lindblad operators (hereafter $|0\rangle$ and $|1\rangle$ identify respectively the excited and the ground state of the single-qubit thermometer). The parameters $\gamma_{-}=\gamma(N+1)$ and $\gamma_{+}=\gamma N$ instead set the temperature dependence of the system dynamics through the Planck number $N=1 /\left(e^{\hbar \omega / k_{B} T}-1\right) \epsilon$ $[0, \infty]$ that counts the average number of resonant bosonic excitations present in the bath, $\gamma$ being a positive rate that fixes the time scale of the problem. By direct integration of the MME, one can easily verify that the state of $\mathcal{S}$ at time $\tau$ can be expressed as $\rho(\tau)=\frac{1}{2}[\mathbb{1}+\vec{r}(\tau) \cdot \vec{\sigma}]$ with a Bloch vector $\vec{r}(\tau)$ having Cartesian components equal to $r_{1,2}(\tau)=r_{1,2}(0) e^{-\gamma(2 N+1) \tau / 2}$ and $r_{3}(\tau)=r_{3}(0) e^{-\gamma(2 N+1) t}-$ $\left(1-e^{-\gamma(2 N+1) \tau}\right) /(2 N+1)$. This corresponds to an evolution induced by generalized amplitude damping (GAD) channels $\Phi_{\tau}$ [39] which, irrespectively from the specific choice of $\rho(0)$, will let the system asymptotically relax to a unique fixed point with the Bloch vector $\vec{r}^{(\mathrm{eq})}=[0,0,-1 /(2 N+1)]$ which represents the system thermal Gibbs state $\rho_{T}^{\text {(eq) }}$. In this long-time limit, our model will behave as anticipated in the previous section, saturating the inequality (3), i.e., allowing one to recover the QCRB via ETF-as well as fulfilling (5). What about the finite-time $\tau$ regime? For the present model the heat capacity $C_{T}(\tau)$ and the energy spread $\Delta^{2} E_{T}(\tau)$ can be easily shown to be equal to

$$
C_{T}(\tau)=\frac{\hbar \omega}{2} \partial_{T} r_{3}(\tau), \quad \Delta^{2} E_{T}(\tau)=\left(\frac{\hbar \omega}{2}\right)^{2}\left[1-r_{3}^{2}(\tau)\right] .
$$

Furthermore, as discussed in the Supplemental Material [36], simple algebra allows us to express the corresponding QFI as

$$
Q_{T}(\tau)=\frac{\left[\partial_{T} r(\tau)\right]^{2}}{1-r^{2}(\tau)}+r^{2}(\tau)\left[\partial_{T} \theta(\tau)\right]^{2},
$$

where $r(\tau)$ and $\theta(\tau)$ are, respectively, the length and the polar angle of the Bloch vector $\vec{r}(\tau)$, the azimuthal angle being a constant of motion and playing no role in the derivation. The first term on the right-hand side of Eq. (7) describes the rearrangement of the population of the probe during its interaction with the reservoir, while the other one accounts for quantum coherence contributions which nullify in the asymptotic limit where $\gamma \tau \rightarrow \infty$ (the first term converging instead to $\left.Q_{T}^{(\mathrm{eq})}\right)$. By direct substitution of these expressions into (3), one can verify that for generic choices of $\tau$ and of the input state $\rho(0)$ the inequality will be strict—see Fig. 1. 

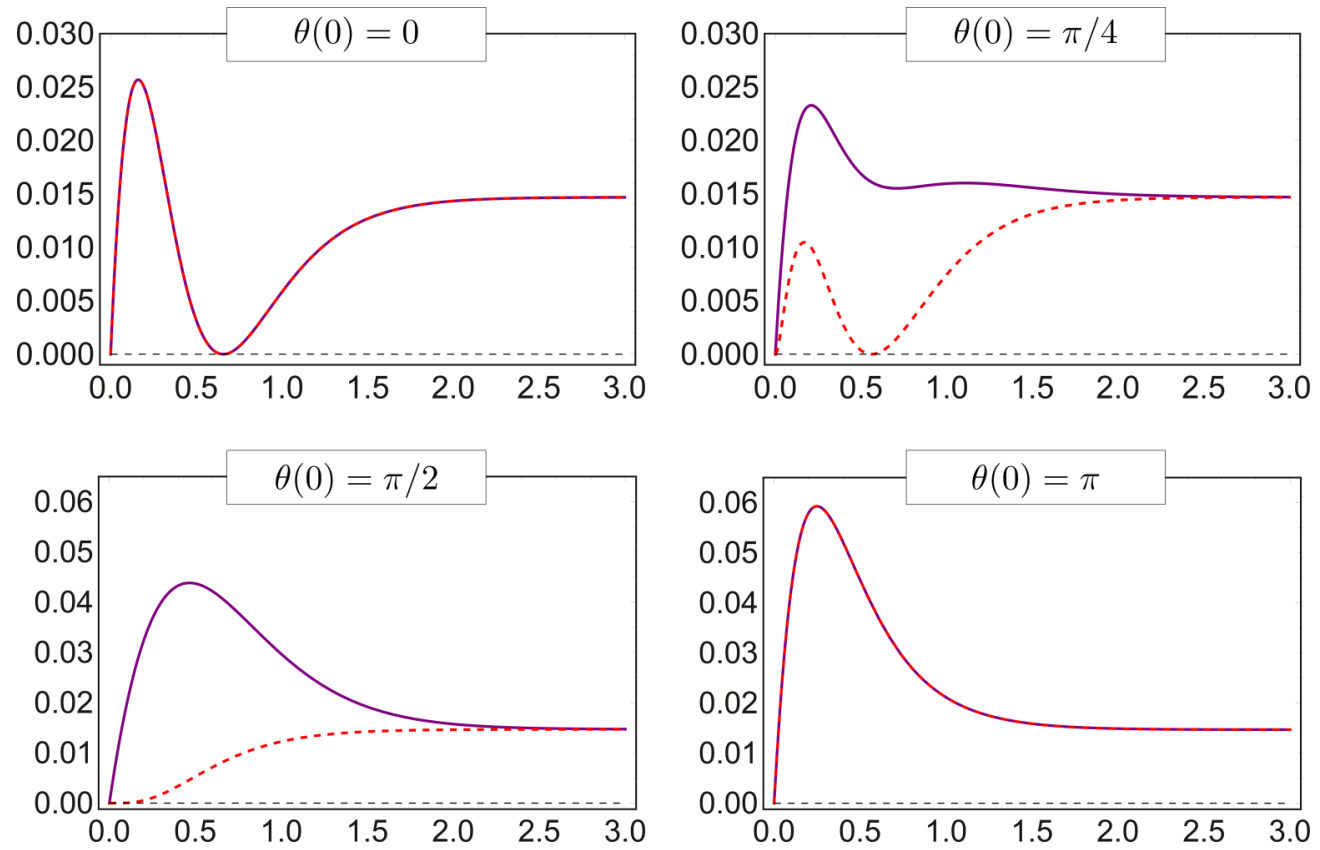

FIG. 1. Plots of the nonequilibrium QFI $Q_{T}(\tau)$ (solid purple curves) and of the quantity $C_{T}^{2}(\tau) / \Delta^{2} E_{T}(\tau)$ (dashed red curves) appearing on the right-hand side of Eq. (3) and which determines the accuracy of the estimation procedure based on direct energy measurement of the probe $\mathcal{S}$. In all plots we assume the input state $\rho(0)$ of $\mathcal{S}$ to be pure with zero azimuthal angle and with the polar angle given by $\theta(0)=0$ (excited state) for the first panel; $\theta(0)=\pi / 4$ for the second; $\theta(0)=\pi / 2$ for the third; and $\theta(0)=0$ (excited state) for the last panel. The temperature $T$ is set equal to 2 in units of $\hbar \omega / k_{B}$ while the time is measured in units of $\gamma^{-1}$. Notice that when the system is initially prepared in a diagonal state, i.e., for $\theta(0)=0$ and $\theta(0)=\pi$, the bound (3) is saturated and the two curves coincide.

A notable exception, however, is obtained when the input state is diagonal into the energy basis of $H$, i.e., when $r_{1,2}(0)$ both nullify [or equivalently when, independently from the choice of $\rho(0)$, the coherence terms of $\rho(\tau)$ are removed by a decoherence process that acts on $\mathcal{S}$ before the measurement stage]. In this special case the system remains diagonal along the full trajectory and Eq. (7) reduces to $Q_{T}(\tau)=\frac{\left[\partial_{T} r_{3}(\tau)\right]^{2}}{1-r_{3}^{2}(\tau)}$. Accordingly, (3) becomes an identity for all choices of the interaction time $\tau$, implying that the standard thermometry scheme which recovers $T$ from just energy measures is optimal. Notice that in this scenario, $\rho(\tau)$ has not reached the thermal equilibrium configuration so ETF arguments cannot be applied: This is made evident by the fact that even though (3) saturates, $Q_{T}(\tau)$ and $C_{T}(\tau)$ cannot yet be linearly connected as in (5) unless one introduces an effective, yet fictitious, rescaling of the proportionality coefficient appearing on the right-hand side. Similar conclusions have been drawn dealing with the role of quantum coherence when thermalization is prevented by a strong coupling between $\mathcal{S}$ and $\mathcal{B}$ [35].

The numerical plots of Fig. 1 show the relations between the left- and right-hand-side terms of (3). In agreement with the finding of Ref. [34], we notice that in general the QFI reaches higher values (corresponding to better estimation accuracies) for finite (possibly dependent on $T$ ) values of $\tau$. Furthermore, after having fixed the parameter $\tau$ at its best, the absolute best performance is obtained when initializing the qubit into the ground state (see the last panel of the figure)we have confirmed this result by numerical optimization of (7), as shown in detail in the Supplemental Material [36].
The first and last panels of Fig. 1 explicitly show the saturation of Eq. (3) for diagonal states at all times $\tau$, while for a generic input this is only possible when $\tau \rightarrow \infty$ since the system asymptotically thermalizes.

\section{QUANTUM PHOTONIC SIMULATION}

We have simulated the evolution of the probing qubit $\mathcal{S}$ under the action of the thermal bath via a photonic implementation of the associated GAD channel $\Phi_{t}$ [40-44], in order to extract the experimental uncertainties on temperature estimation. For this purpose we have exploited the Kraus representation of the map $\rho(\tau)=\Phi_{\tau}[\rho(0)]=$ $\sum_{i=1}^{4} K_{i} \rho(0) K_{i}^{\dagger}$, where $K_{i}$ 's are four Kraus operators: The first two, i.e., $K_{1}=\sqrt{\frac{1+N}{1+2 N}}\left(e^{-\gamma(2 N+1) \tau / 2}|0\rangle\langle 0|+| 1\rangle\langle 1|\right)$, $K_{2}=\sqrt{\frac{1+N}{1+2 N}} \sqrt{1-e^{-\gamma(2 N+1) \tau}}|1\rangle\langle 0|$ being responsible for decay from the excited $|0\rangle$ to the ground $|1\rangle$ state, represent the action of an amplitude damping (AD) map, and the second two, i.e., $K_{3}=\sqrt{\frac{N}{2 N+1}}\left(|0\rangle\left\langle 0\left|+e^{-\gamma(2 N+1) \tau / 2}\right| 1\right\rangle\langle 1|\right)$, $K_{4}=\sqrt{\frac{N}{2 N+1}} \sqrt{1-e^{-\gamma(2 N+1) \tau}}|0\rangle\langle 1|$, describing the absorption events, represent instead an inverse amplitude damping (IAD) map. The previous decomposition depicts the GAD as a weighted sum of two different processes, an $\mathrm{AD}$ and an IAD with weights respectively equal to $\sqrt{\frac{N+1}{2 N+1}}$ and $\sqrt{\frac{N}{2 N+1}}$. This last property is crucial for implementing a quantum optical simulation of the process: After reproducing the AD and the IAD channel through a succession of optical logic 

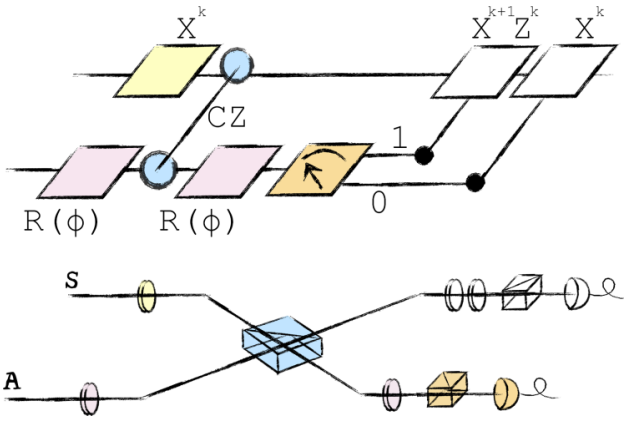

FIG. 2. Quantum simulation via quantum photonics. Upper panel: Quantum circuits for the implementation of the $\mathrm{AD}(k=0)$, and of the IAD $(k=1)$ channels [41]. The circuital elements are as follows: $\mathrm{X}$ and $\mathrm{Z}$, that implement the Pauli rotations $\sigma_{x}$ and $\sigma_{z}$; $\mathrm{CZ}$, representing a controlled- $\sigma_{z}$ gate; $R(\phi)$ is a rotation by an angle $\phi$ around the $y$ axis. The measurements are performed in the computational basis. Lower panel: Experimental scheme. One photon is employed to simulate the single-qubit thermometer, while a second one is used as an ancilla to simulate the system-bath interaction. The computational basis is encoded in the vertical and the horizontal polarizations of the single photons. The single-qubit gates in the upper panel are implemented by means of half-wave plates (HWPs), while the $\mathrm{CZ}$ is realized by a partially polarizing beam splitter. More details are given in the Supplemental Material [36].

gates, it is possible to reconstruct the full density matrix simply by doing a proper weighted sum of the outputs of the two channels, as depicted in Fig. 2 [28]. Specifically, an $\mathrm{AD}$ acting on a qubit $\mathcal{S}$ can be formally simulated by coupling the system with an ancilla $\mathcal{A}$ and doing the following operations:

(1) A controlled- $\sigma_{z}$ gate, with $\mathcal{S}$ as the control, embedded between two rotations $R(\phi)$ acting on $\mathcal{A}$ [45-47]. The rotations are performed around the $y$ axis and the angle $\phi$ has to be chosen in order to mimic the damping factor of the Kraus decomposition of the map, and in our case it is such that $e^{-\gamma(2 N+1) \tau}=\cos ^{2}(2 \phi)[28,48]$.

(2) A projective measurement on the computational basis of $\mathcal{A}$, conditioning a $\sigma_{x}$ gate on $\mathcal{S}$ (see Fig. 2).
The above-mentioned procedure works also for the IAD, except for two additional $\sigma_{x}$ and $\sigma_{z}$ rotations in the preparation and postprocessing of the state. An experimental implementation is obtained by associating each logical gate with its corresponding element in the optical table, as explained in Fig. 2.

The mean value of the energy and the temperature uncertainty are inferred by performing a measure on the Hamiltonian eigenbasis of $\mathcal{S}$, a purpose that in practice is realized through experimental counts of the populations [49]. For any simulated time in the experiment, the expectation value of the energy is given by $\langle E\rangle=\left(n_{0}-n_{1}\right) / 2\left(n_{0}+n_{1}\right)$, where $n_{i}$ corresponds to the measured count rate of the state $i$. Its uncertainty is evaluated as $\Delta^{2} E=n_{0} n_{1} /\left(n_{0}+n_{1}\right)^{3}$; this is an estimation of the energy spread $\Delta^{2} E_{T}(\tau)$. Temperature uncertainties (at each estimation round) are then obtained as $\Delta^{2} T=\Delta^{2} E /\left(\partial_{T} E\right)^{2}$. The results are summarized in Fig. 3, in which we compare the experimental uncertainties on the temperature with the related QCRB.

\section{v. CONCLUSIONS}

At thermal equilibrium, the ETF establishes a neat link between the temperature fluctuations $\Delta T$, and the thermal susceptibility of the system corresponding to the heat capacity. We have investigated if similar relations can be recovered in nonequilibrium regimes. For a single-qubit thermometer, we have shown this is not possible when coherence is present in the initial state of the probe: The QFI functional gauging the optimal accuracy threshold contains additional contributions. However, for diagonal input states the optimality of standard measurement procedure is restored and allows one to saturate the QCRB with conventional thermometry based on energy measurements. This peculiar effect is probably related with the small number of degrees of freedom characterizing the thermometer we used. We suspect that as the dimensionality of the probing system increases, optimal thermometry could only be achieved by more complex measurement procedures which, even in the absence of off-diagonal terms, include the study of the full statistic of the energy measures.
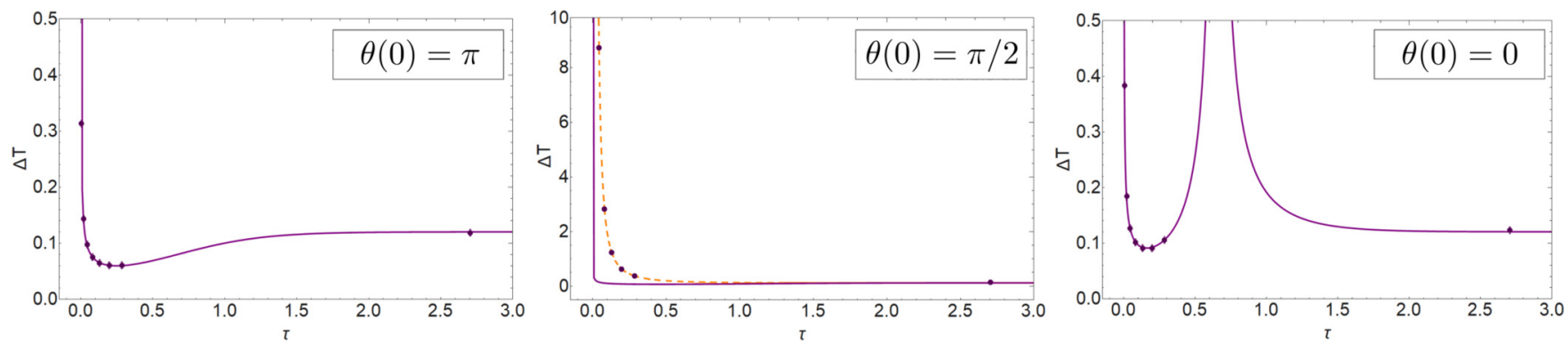

FIG. 3. Comparison between the experimental errors $\Delta T$, and metrological figures of merit related to the temperature parameter. In the three panels, the solid purple curves represent the theoretical QCRB, the dashed orange curve represents the theoretical CRB, and the purple points represent the experimental uncertainties on the temperature. In the left panel, we confirm that the ground state allows one to reach the greatest sensitivity of the single-qubit probe as it permits to reach the lowest value of $\Delta T$; in the right panel, we show the behavior of the probe prepared in the excited state, and we observe a divergence in the QCRB due to the presence of a zero in the QFI-see the first panel of Fig. 1; in the middle panel, we show the coherent strategy. Here, the experimental uncertainties on the temperature do not reach the QCRB but they are well captured by its classical counterpart. 


\section{ACKNOWLEDGMENTS}

A.D.P. acknowledges financial support from the University of Florence in the framework of the University Strategic Project Program 2015 (Project No. BRS00215). This project has received funding from the European Commission Horizon 2020 research and innovation programme under Grant Agreement No. 665148 (QCUMbER).
[1] V. Giovannetti, S. Lloyd, and L. Maccone, Phys. Rev. Lett. 96, 010401 (2006).

[2] V. Giovannetti, S. Lloyd, and L. Maccone, Nat. Photon. 5, 222 (2011).

[3] M. G. A. Paris, Int. J. Quantum Inf. 7, 125 (2009).

[4] S. Hilt and E. Lutz, Phys. Rev. A 79, 010101(R) (2009).

[5] N. S. Williams, K. Le Hur, and A. N. Jordan, J. Phys. A: Math. Theor. 44, 385003 (2011).

[6] M. Kliesch, C. Gogolin, M. J. Kastoryano, A. Riera, and J. Eisert, Phys. Rev. X 4, 031019 (2014).

[7] S. Vinjanampathy and J. Anders, Contemp. Phys. 57, 545 (2016).

[8] J. Millen and A. Xuereb, New J. Phys. 18, 011002 (2016).

[9] P. J. Mohr and B. N. Taylor, Rev. Mod. Phys. 77, 1 (2005).

[10] W. Weng, J. D. Anstie, T. M. Stace, G. Campbell, F. N. Baynes, and A. N. Luiten, Phys. Rev. Lett. 112, 160801 (2014).

[11] T. M. Stace, Phys. Rev. A 82, 011611(R) (2010).

[12] R. B. Mann and E. Martin-Martinez, Found. Phys. 44, 492 (2014).

[13] M. Mehboudi, M. Moreno-Cardoner, G. De Chiara, and A. Sanpera, New J. Phys. 17, 055020 (2015).

[14] A. De Pasquale, D. Rossini, R. Fazio, and V. Giovannetti, Nat. Commun. 7, 12782 (2016).

[15] A. De Pasquale, K. Yuasa, and V. Giovannetti, Phys. Rev. A 96, 012316 (2017).

[16] G. De Palma, A. De Pasquale, and V. Giovannetti, Phys. Rev. A 95, 052115 (2017).

[17] S. Campbell, M. Mehboudi, G. De Chiara, and M. Paternostro, New J. Phys. 19, 103003 (2017).

[18] M. Brunelli, S. Olivares, and M. G. A. Paris, Phys. Rev. A 84, 032105 (2011).

[19] M. Brunelli, S. Olivares, M. Paternostro, and M. G. A. Paris, Phys. Rev. A 86, 012125 (2012).

[20] C. Sabín, A. White, L. Hackermuller, and I. Fuentes, Sci. Rep. 4, 6436 (2014).

[21] T. H. Johnson, F. Cosco, M. T. Mitchison, D. Jaksch, and S. R. Clark, Phys. Rev. A 93, 053619 (2016).

[22] M. Hohmann, F. Kindermann, T. Lausch, D. Mayer, F. Schmidt, and A. Widera, Phys. Rev. A 93, 043607 (2016).

[23] U. Marzolino and D. Braun, Phys. Rev. A 88, 063609 (2013).

[24] L. A. Correa, M. Perarnau-Llobet, K. V. Hovhannisyan, S. Hernandez-Santana, M. Mehboudi, and A. Sanpera, Phys. Rev. A 96, 062103 (2017).

[25] G. Salvatori, A. Mandarino, and M. G. A. Paris, Phys. Rev. A 90, 022111 (2014).

[26] S. Jevtic, D. Newman, T. Rudolph, and T. M. Stace, Phys. Rev. A 91, 012331 (2015).

[27] W. K. Tham, H. Ferretti, A. V. Sadashivan, and A. M. Steinberg, Sci. Rep. 6, 38822 (2016).

[28] L. Mancino, M. Sbroscia, I. Gianani, E. Roccia, and M. Barbieri, Phys. Rev. Lett. 118, 130502 (2017).
[29] S. Campbell, M. G. Genoni, and S. Deffner, Quantum Sci. Technol. 3, 025002 (2018).

[30] L. D. Landau and E. M. Lifshitz, Statistical Physics: Part I (Butterworth-Heinemann, Oxford, UK, 1980).

[31] M. Falcioni, D. Vallamaina, A. Vulpiani, A. Puglisi, and A. Sarracino, Am. J. Phys. 79, 777 (2011).

[32] C. Di Castro and R. Raimondi, Statistical Mechanics and Applications in Condensed Matter (Cambridge University Press, Cambridge, UK, 2015).

[33] A. Puglisi, A. Sarracino, and A. Vulpiani, Phys. Rep. 709-710, 1 (2017).

[34] L. A. Correa, M. Mehboudi, G. Adesso, and A. Sanpera, Phys. Rev. Lett. 114, 220405 (2015).

[35] H. J. D. Miller and J. Anders, Nat. Commun. 9, 2203 (2018).

[36] See Supplemental Material at http://link.aps.org/supplemental/ 10.1103/PhysRevA.98.050101 for detailed calculations and the description of the experimental apparatus.

[37] P. Zanardi, P. Giorda, and M. Cozzini, Phys. Rev. Lett. 99, 100603 (2007).

[38] P. Zanardi, M. G. A. Paris, and L. Campos Venuti, Phys. Rev. A 78, 042105 (2008).

[39] M. A. Nielsen and I. L. Chuang, Quantum Computations and Quantum Information (Cambridge University Press, Cambridge, UK, 2000).

[40] D.-S. Wang, D. W. Berry, M. C. de Oliveira, and B. C. Sanders, Phys. Rev. Lett. 111, 130504 (2013).

[41] H. Lu, C. Liu, D.-S. Wang, L.-K. Chen, Z.-D. Li, X.-C. Yao, L. Li, N.-L. Liu, C.-Z. Peng, B. C. Sanders, Y.-A. Chen, and J.-W. Pan, Phys. Rev. A 95, 042310 (2017).

[42] A. Aspuru-Guzik and P. Walther, Nat. Phys. 8, 285 (2012).

[43] S. Cialdi, M. A. Rossi, C. Benedetti, B. Vacchini, D. Tamascelli, S. Olivares, and M. G. Paris, Appl. Phys. Lett. 110, 081107 (2017).

[44] A. Crespi, S. Longhi, and R. Osellame, Phys. Rev. Lett. 108, 163601 (2012).

[45] N. K. Langford, T. J. Weinhold, R. Prevedel, K. J. Resch, A. Gilchrist, J. L. O’Brien, G. J. Pryde, and A. G. White, Phys. Rev. Lett. 95, 210504 (2005).

[46] N. Kiesel, C. Schmid, U. Weber, R. Ursin, and H. Weinfurter, Phys. Rev. Lett. 95, 210505 (2005).

[47] R. Okamoto, H. F. Hofmann, S. Takeuchi, and K. Sasaki, Phys. Rev. Lett. 95, 210506 (2005).

[48] L. Mancino, V. Cavina, A. De Pasquale, M. Sbroscia, R. I. Booth, E. Roccia, I. Gianani, V. Giovannetti, and M. Barbieri, Phys. Rev. Lett. 121, 160602 (2018).

[49] The global count rates of the process are obtained combining those measured in the two subprocesses, viz., the simulated AD and IAD channels. The weighting procedure to simulate the output of the GAD is done $a b$ initio by using each channel a proper number of times. 